\title{
Estudo clínico comparativo de três doses de atracúrio em cães (Canis familliaris)
}

\author{
Clinical comparative study of three doses of atracurium in dogs \\ (Canis familliaris)
}

\author{
Thomas Becker, ${ }^{\star}$ Firmino Marsico Filho, ${ }^{\star \star}$ Marcelo da Rocha Kastrup, ${ }^{\star}$ Paulo Roberto Loureiro do Nascimento, **

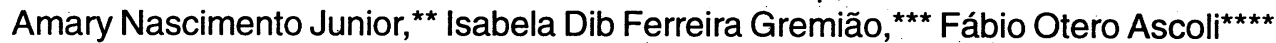

\begin{abstract}
Resumo
Foram utilizados seis cães, sem raça definida, com peso variando entre 8 e $25 \mathrm{~kg}$. Cada animal foi avaliado três vezes, com intervalo de uma semana entre os experimentos. Como medicação pré-anestésica, empregou-se o maleato de acepromazina $0,1 \mathrm{mg} / \mathrm{kg}$ IM) e como agente indutor e de manutenção, propofol nas doses $(4,0 \mathrm{mg} / \mathrm{kg} \mathrm{EV})$ e $(0,4 \mathrm{mg} / \mathrm{kg} / \mathrm{min} \mathrm{EV})$ respecti/amente. A função neuromuscular foi monitorizada através de acelerometria, utilizando-se uma seqüência de quatro estímuos (TOF) com intervalos de 15 segundos aplicada no nervo fibular comum. Após a estabilização da altura da resposta aos estímulos, foram administradas doses de $0,1,0,2$ e 0,3mg/kg EV, que correspondem, respectivamente, a 1, 2 e 3 vezes a $\mathrm{DE}_{90}$ do atracúrio. Foram avaliados os seguintes parâmetros: tempo de instalação do bloqueio máximo, grau de bloqueio neuromuscular, tempo de relaxamento cirúrgico e tempo de restabelecimento da transmissão neuromuscular. Foram encontradas diferenças estatisticamente significativas $(\mathrm{P}<0,05)$ entre as doses de atracúrio administradas para todos os parâmetros avaliados. Concluiu-se que, doses de 0,2 e $0,3 \mathrm{mg} / \mathrm{kg}$ de atracúrio em cães induzidos e mantidos sob infusão contínua de propofol proporcionaram um relaxamento adequado para intervenções cirúrgicas que necessitem de relaxamento muscular.
\end{abstract}

Palavras-chave: bloqueador neuromuscular; atracúrio; cão.

\section{Introdução}

Os agentes bloqueadores neuromusculares são mais freqüentemente utilizados como adjuvantes da anestesia geral em pacientes humanos e empregados rotineiramente com a finalidade de facilitar a intubação endotraqueal (Taylor, 1996). Na prática anestesiológica veterinária, estes agentes são pouco utilizados, devido ao fato de a maioria dos animais permitir a intubação endotraqueal sob anestesia . geral (Cullen, 1996). No entanto, seu emprego está indicado em vários procedimentos, como: nas laparatomias, oferecendo relaxamento muscular abdominal; nas toracotomias, onde é imprescindível uma ventilação pressão positiva intermitente; nas manipulações ortopédicas; e como um dos componentes na técnica de anestesia balanceada (Klein, 1987; Booth, 1992; McKelvy e Hollingshead, 1994; Cullen, 1996; Taylor, 1996).

O atracúrio é um bloqueador neuromuscular adespolarizante de duração intermediária. Em virtude de sua degradação espontânea no sangue, praticamente independente da biotransformação visceral, oferece um aspecto de segurança maior em relação a outros bloqueadores. Este fármaco é particularmente útil em pacientes com insuficiência renal, hepática e em cirurgias de transplante.

Recentemente, um novo grupo farmacológico denominado fenólicos tornou-se uma nova opção com agente indutor è de manutenção anestésica devido a apresentar algumas vantagens como: ausência de lesões tissulares quando do seu extravasamento do leito vascular e rápido retorno das atividades motoras (Glen et al., 1985; Mama \& Steffey, 1995), além de abolir a potencialização do efeito miorrelante dos bloqueadores neuromusculares adespolarizantes, observado quando da utilização de agentes anestésicos voláteis (Marshall \& Longnecker, 1996).

O objetivo do presente trabalho é comparar clinicamente em cães a resposta após injeção única de atracúrio em três doses $(0,1 ; 0,2$ e $0,3 \mathrm{mg} / \mathrm{kg} \mathrm{EV})$, avaliando-se o tempo de instalação do bloqueio máximo, tempo de relaxamento cirúrgico, tempo de restabelecimento da transmissão neuromuscular, observando a capacidade de cada dose de atracúrio proporcionar relaxamento cirúrgico adequado em cães.

\footnotetext{
* Alunos de Pós- graduação em Medicina Veterinária - UFF.

** Professores de Faculdade de Veterinária (UFF) * Pesquisador CNPq. Rua Vital Brazil Filho, 64 - Vital Brazil, Niterói, RJ - CEP: $24230-340$.

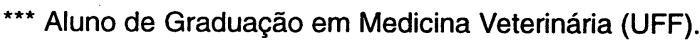

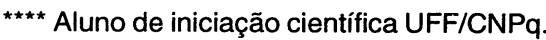




\section{Material e métodos}

Foram utilizados seis cães, fêmeas, sem raça definida (SRD), na faixa etária de 2 a 3 anos, hígidos, com peso corporal variando entre 8 a $25 \mathrm{~kg}$. Os animais receberam manejo padronizado e, antes dos experimentos, foram submetidos ao exame clínico, laboratorial e hemogasométrico, ${ }^{1}$ sendo observado jejum alimentar de 12 e hídrico de 3 horas. Cada animal foi avaliado três vezes de forma randomizada, com intervalo de uma semana entre os experimentos. Após pré-medicação com maleato de acepromazina ${ }^{2}(0,1 \mathrm{mg} / \mathrm{kg} \mathrm{IM})$, os animais foram induzidos com propofol ${ }^{3}(4,0 \mathrm{mg} / \mathrm{kg} \mathrm{EV})$ lentamente até a obtenção do relaxamento da musculatura masseteriana, intubados, conectados a um circuito semi-aberto ${ }^{4} \mathrm{e}$ posicionados em decúbito lateral direito sobre um colchão térmico. ${ }^{5}$ A manutenção anestésica foi realizada com a administração contínua de propofol na velocidade de 0,4 $\mathrm{mg} / \mathrm{kg} / \mathrm{min}$ através de bomba de infusão contínua, ${ }^{6}$ seguida da monitorização da frequencia cardíaca, ${ }^{7}$ pressão arterial não-invasiva ${ }^{8}$ e grau de saturação pelo $\mathrm{O}_{2}{ }^{9}$

Para a monitorização da transmissão neuromuscular, empregou-se um acelerômetro. ${ }^{10}$ Foi realizada a tricotomia ampla e rigorosa da face lateral da região da coxa e a área que cobre o músculo tibial cranial do membro esquerdo. Estas superfícies foram desengorduradas com éter sulfúrico com a finalidade de permitir a fixação dos eletrodos, do transdutor de aceleração e do sensor de temperatura.

Dois eletrodos de $\mathrm{Ag} / \mathrm{AgCl}^{11} \mathrm{com}$ adesivo condutor foram posicionados sobre a superfície que correspondente ao nervo fibular comum. O transdutor de aceleração foi fixado com esparadrapo sobre a área que cobre o músculo tibial cranial e o sensor de temperatura próximo aos eletrodos. A região társica do membro posterior esquerdo foi imobilizado com esparadrapo na mesa cirúrgica. A corrente supramáxima foi ajustada e a resposta ao estímulo para $100 \%$ num procedimento automático do acelerômetro. A seqüência de quatro estímulos (TOF) com intervalo de 15 segundos foi utilizada para a monitorização da transmissão neuromuscular. Após estabilização da altura da resposta, foi efetuada a injeção rápida do atracúrio ${ }^{12}(0,1 ; 0,2$ ou $0,3 \mathrm{mg} / \mathrm{kg}$ ) por via endovenosa. Após a administração do miorrelaxante, a respiração dos cães foi assistida e, no caso de apnéia, controlada manualmente.

Foram avaliados os seguintes parâmetros: tempo de instalação do bloqueio máximo $\left(T_{\max }\right)$, valor do bloqueio máximo (Bloqueio ${ }_{\max }$ ), tempo de restabelecimento do T1 para $25 \%$ do controle (DUR ${ }_{25}$ ), tempo de restabelecimento do T1 para $75 \%$ do controle $\left(D U R_{75}\right)$, tempo de restabelecimento da razão de TOF para $70 \%$ do controle
$\left(D_{\mathrm{TUF}=70}\right)$ e período de relaxamento cirúrgico $(\mathrm{RC})$ que corresponde ao período quando T1 está menor ou igual a $10 \%$ do controle.

Os resultados obtidos das mensurações dos vários parâmetros foram submetidos ao teste não-paramétrico de Wilcoxon $(p<0,05)$.

\section{Resultado}

Os resultados da administração de atracúrio nas doses de 0,$1 ; 0,2$ e $0,3 \mathrm{mg} / \mathrm{kg}$ estão apresentados nas tabelas 1 e 2 .

Com aumento na dose do atracúrio, houve redução significativa no tempo de instalação do bloqueio máximo de $4,33 \pm 0,88$ minutos para a dose de $0,1 \mathrm{mg} / \mathrm{kg}, 3,17 \pm 1,78$ minutos para $0,2 \mathrm{mg} / \mathrm{kg}$ e 2,21 $\pm 0,77$ minutos para 0,3 mg/kg.

Em relação ao bloqueio, observou-se aumento no grau com doses crescentes do miorrelaxante. Na dose mais baixa, o grau de bloqueio alcançado foi de $84,83 \pm 9,26 \%$. Já nas doses de 0,2 e $0,3 \mathrm{mg} / \mathrm{kg}$, foram de $95,60 \pm 3,06 \%$ e $99,5 \pm 1,22 \%$, respectivamente.

Encontramos um aumento $(p<0.05)$ na duração de ação do miorrelaxante com aumento da dose. Para a dose de $0,1 \mathrm{mg} / \mathrm{kg}$, o DUR 25 foi de $11,31 \pm 2,74$ minutos, para 0,2 $\mathrm{mg} / \mathrm{kg}$, de 16,25 $\pm 5,22$ minutos e para $0,3 \mathrm{mg} / \mathrm{kg}, 23,79 \pm 3,34$ minutos. Em relação ao $\mathrm{DUR}_{75}$, encontrou-se resultado semelhante, $13,04 \pm 3,77$ minutos, $20,75 \pm 5,93$ e $30,17 \pm 2,72$ minutos para as doses de $0,1,0,2$ e $0,3 \mathrm{mg} / \mathrm{kg}$, respectivamente. O DUR $\mathrm{TOF}=70$ para as três doses em ordem crescente foi de $16,21 \pm 5,66,26,25 \pm 6,02$ e $37,67 \pm 5,10$ minutos, respectivamente.

A duração do período de relaxamento cirúrgico foi maior com aumento na dose do atracúrio. O relaxamento cirúrgico para a dose de $0,1 \mathrm{mg} / \mathrm{kg}$ apresentou grande variação e foi de 2,21 $\pm 3,59$ minutos. Nas doses de 0,2 e $0,3 \mathrm{mg} / \mathrm{kg}$, 0 tempo foi bem maior com 12,42 $\pm 5,34$ e 20,17 $\pm 2,47$ minutos, respectivamente.

Tabela 1: Parâmetros relativos ao início e duração do atracúrio após administração de três doses $(0,1 ; 0,2$ e 0,3 $\mathrm{mg} / \mathrm{kg}$ ) em seis cães. Letras diferentes na mesma coluna apresentam diferenças estatisticamente significativas pelo teste de Wilcoxon $(p<0,05)$.

\begin{tabular}{c|c|c|c|c}
\hline $\begin{array}{c}\text { Dose } \\
(\mathrm{mg} / \mathrm{kg})\end{array}$ & $\begin{array}{c}\mathrm{T}_{\max } \\
(\mathrm{min})\end{array}$ & $\begin{array}{c}\mathrm{DUR}_{25} \\
(\mathrm{~min})\end{array}$ & $\begin{array}{c}\mathrm{DUR}_{75} \\
(\mathrm{~min})\end{array}$ & $\begin{array}{c}\text { DUR }_{\text {TOF }}=70 \\
(\mathrm{~min})\end{array}$ \\
\hline 0,10 & $4,33 \pm 0,88_{\mathrm{A}}$ & $11,31 \pm 2,74_{\mathrm{A}}$ & $13,04 \pm 3,77_{\mathrm{A}}$ & $16,21 \pm 5,66_{\mathrm{A}}$ \\
\hline 0,20 & $3,17_{1} 1,78_{\mathrm{B}}$ & $16,25 \pm 5,22_{\mathrm{B}}$ & $20,75 \pm 5,93_{\mathrm{B}}$ & $26,25 \pm 6,02_{\mathrm{B}}$ \\
\hline 0,30 & $2,21 \pm 0,77_{\mathrm{C}}$ & $23,79_{ \pm} 3,34_{\mathrm{C}}$ & $30,17 \pm 2,72_{\mathrm{C}}$ & $37,67 \pm 5,10_{\mathrm{C}}$ \\
\hline
\end{tabular}

'Drake Ags - 21; ${ }^{2}$ Acepran 1\% - Lab. Univet; ${ }^{3}$ Diprivan - Lab. Zeneca; ${ }^{4} \mathrm{KTT}$ - Baraka; ${ }^{5}$ Vetko Thermal Barrier - Model noV-20 s/n 5020; ${ }^{6} \mathrm{Digipump}$ SR2000 - Digicare; ${ }^{7}$ Monitor cardiaco - am78100A ECG monitor - anamed; ${ }^{8}$ Dixtal DX2710; ${ }^{9}$ Nellcor Pulse Oximeter; ${ }^{10}$ TOF-GUARD Organon Teknika NV; "Meditrace 200 - Graphic Controls; ${ }^{12}$ Tracrium - Lab. Wellcome - Zeneca 
Tabela 2: Valores médios para período de relaxamento cirúrgico e de bloqueio máximo, após administração três doses $(0,1 ; 0,2$ e $0,3 \mathrm{mg} / \mathrm{kg})$ em seis cães. Letras diferentes na mesma coluna apresentam diferenças estatisticamente significativas pelo teste de Wilcoxon $(p<0,05)$.

\begin{tabular}{c|c|c}
\hline $\begin{array}{c}\text { Dose } \\
(\mathrm{mg} / \mathrm{kg})\end{array}$ & $\begin{array}{c}\mathrm{RC} \\
(\mathrm{min})\end{array}$ & $\begin{array}{c}\text { Bloqueio } \\
(\%)\end{array}$ \\
\hline 0,10 & $2,21 \pm 3,59_{\mathrm{A}}$ & $84,83 \pm 9,26_{\mathrm{A}}$ \\
\hline 0,20 & $12,42 \pm 5,34_{\mathrm{B}}$ & $12,42 \pm 5,34_{\mathrm{B}}$ \\
\hline 0,30 & $20,17 \pm 2,47_{\mathrm{C}}$ & $20,17 \pm 2,47_{\mathrm{C}}$ \\
\hline
\end{tabular}

\section{Discussão}

A dosagem do atracúrio em cães descrita na literatura é de 0,2 a 0,4 mg/kg (Cullen, 1996). Em nosso estudo, utilizamos a DE90 em cães que corresponde respectivamente a $0,1 \mathrm{mg} / \mathrm{kg}$ (Hughes e Chapple, 1981), 2 x DE90 e 3 x DE90.

Segundo observações de Jones et al. (1983), em que oito cães foram mantidos sob anestesia geral com tiopental sódico e óxido nitroso, doses de 0,2; 0,4 e 0,6 $\mathrm{mg} / \mathrm{kg}$ de atracúrio apresentaram tempos de instalação do bloqueio máximo respectivamente de 2,7; 1,65 e 1,54 minutos. Hughes \& Chapple (1981), utilizando quatro cães mantidos sob anestesia geral com alfa-cloralose, encontraram $T_{\max }$ de $4,9( \pm 0,8) ; 2,3( \pm 0,3)$ e $1,8( \pm 0,3)$ minutos para as doses de 0,$125 ; 0,25$ e $0,5 \mathrm{mg} / \mathrm{kg}$, respectivamente, com a utilização do padrão de estimulação isolado. Já com o emprego da estimulação tetânica a $30 \mathrm{~Hz}$, estes autores encontraram valores de $3,49( \pm 0,9) ; 1,0$ $( \pm 0,2)$ e $1,1( \pm 0,2)$ minutos. A redução gradativa do $T_{\max }$ com doses crescentes do bloqueador, também foi constatada em nosso experimento em que as doses de 0,1 ; 0,2 e $0,3 \mathrm{mg} / \mathrm{kg}$ de atracúrio corresponderam a $T_{\text {max }}$ de $4,33( \pm 0,88) ; 3,17( \pm 1,78)$ e 2,21 $( \pm 0,77)$ minutos. A diferença observada nos valores de $T_{\max }$ pode ser devido a utilização da mecanomiografia como método de monitorização, além da estimulação do nervo ulnar no experimento realizado por Jones et al. (1983). Por outro lado, Hughes \& Chapple (1981) empregaram padrões de estimulação diferentes, além de terem utilizado a mecanomiografia.

Os valores de bloqueio máximo obtidos por Hughes \& Chapple (1981), com as doses de 0,25 e 0,5 mg/kg de atracúrio, foram de $97( \pm 2) \%$ e $100 \%$, respectivamente, utilizando o padrão de estimulação isolado. Em nosso experimento, a dose de $0,1 \mathrm{mg} / \mathrm{kg}$ alcançou um Bloqueio $_{\max }$ de $84,83( \pm 9,26) \%$, a dose de $0,2 \mathrm{mg} / \mathrm{kg}$, $95,6( \pm 3,06) \%$ e a dose de $0,3 \mathrm{mg} / \mathrm{kg}, 99,5( \pm 1,22) \%$ $(p<0,05)$.

Quanto aos valores relativos ao restabelecimento do bloqueio neuromuscular, encontramos um aumento na duração de ação do atracúrio proporcional ao aumento da dose empregada, conforme o observado por Hughes \& Chapple (1981). Não há registros referentes a valores de razão de TOF relativo ao atracúrio em Anestesiologia Veterinária. Em nosso experimento, encontramos diferenças estatisticamente significativas para DUR ${ }_{\mathrm{TOF}=70}$ entre as doses de atracúrio utilizadas $(p<0,05)$. Segundo Brand \& Cullen. (1977), uma razão de TOF de $70 \%$ está relacionada a sinais clínicos de recuperação do bloqueio neuromuscular em pacientes humanos como: capacidade de manter os olhos abertos, capacidade de segurar com as mãos, protrusão de língua e esforço respiratório adequado para serem extubados. Observamos que quando a razão de TOF alcançava $70 \%$, todos os cães apresentavam esforço respiratório satisfatório. No entanto, devido à impossibilidade de avaliar os demais parâmetros que dependem da cooperação ativa dos animais de experimento e devido à recuperação destes dos efeitos anestesiológicos, não pudemos determinar 0 momento exato da extubação.

Jones (1992) sugere um bloqueio de pelo menos $75 \%$ para que haja um relaxamento cirúrgico aceitável. Em nosso experimento, utilizamos um bloqueio igual ou maior que $90 \%$ para determinar o período de relaxamento cirúrgico (RC). Quando empregamos a dose de $0,1 \mathrm{mg} / \mathrm{kg}$ de atracúrio, apenas $50 \%$ dos animais apresentaram relaxamento adequado e com as doses de 0,2 e $0,3 \mathrm{mg} / \mathrm{kg}$, proporcionaram um relaxamento cirúrgico de $12,42( \pm 5,34)$ e $20,17( \pm 2,47)$ minutos, respectivamente, em $100 \%$ dos animais.

A utilização do propofol como agente anestésico para a manutenção da anestesia geral em nosso experimento foi com o intuito de abolir a potencialização do efeito miorrelaxante dos bloqueadores neuromusculares adespolarizantes, observado quando da utilização de agentes anestésicos voláteis (Marshall \& Longnecker, 1996). Desta forma, acreditamos que o número de animais que alcançaram o período de relaxamento cirúrgico nestas condições, poderia ter sido maior nas doses utilizadas, caso tivéssemos utilizado um agente anestésico volátil (Goudsouzian \& Parsloe, 1994).

\section{Conclusões}

A dosagem de $0,1 \mathrm{mg} / \mathrm{kg}$ de atracúrio em cães, após indução e manutenção sob infusão contínua com propofol, mostrou-se insuficiente para proporcionar relaxamento cirúrgico adequado. Já as doses de 0,2 e 0,3 mg/kg do miorrelaxante ofereceram relaxamento adequado, podendo ser utilizadas em procedimentos cirúrgicos que requerem bom relaxamento.

Com o aumento na dose do atracúrio administrado, houve redução no tempo de instalação do bloqueio máximo e aumento na duração de ação do miorrelaxante nas doses empregadas. As diferenças entre as doses foram estatisticamente significativas. $(p<0,05)$. 


\begin{abstract}
Six mongrel dogs weighting 8 to $25 \mathrm{~kg}$ were included in this study. Each animal was acessed three times with a seven day interval between each experiment. After the dogs were premedicated $(0,1 \mathrm{mg} / \mathrm{kg} \mathrm{IM})$ with acepromazine maleate, anesthesia was induced $(4,0 \mathrm{mg} / \mathrm{kg})$ and maintained with a continuous infusion $(0,4 \mathrm{mg} / \mathrm{kg} / \mathrm{min})$ of propofol. Train-of-four stimuli repeated at 15 seconds interval were applied on the fibular nerve and the neuromuscular transmission monitorized by accelerometry. After stabilization of the twitch height, 0,$1 ; 0,2$ and $0,3 \mathrm{mg} / \mathrm{kg}$ of atracurium was administered intravenously for each group, which correspond to 1,2 and 3 times $\mathrm{ED}_{90}$ of the neuromuscular blocker. In this study, we evaluated the onset time, neuromuscular block, period of surgical relaxment and the time for recovery from neuromuscular block. Significant differences due to diferent dosis of atracurium were found for all parameters. We concluded that atracurium at dosis of 0,2 and $0,3 \mathrm{mg} / \mathrm{kg}$, in dogs induced and maintained with a continuous infusion of propofol, provides appropriate relaxment for surgical access.
\end{abstract}

Keywords: neuromuscular block; atracurium; dog.

\section{Referências bibliográficas}

BOOTH, N. H., MCDONALD, L. E. Farmacologia colinérgica: agentes bloqueadores neuromusculares, p.110-120. In: Farmacologia e Terapêutica em Veterinária, 6. ed., Guanabara Koogan, Rio de Janeiro, 1992.

BRAND, J. B., CULLEN, D. J. Spontaneous recovery from nondepolarizing neuromuscular blockade: correlation between clinical and evoked responses. Anesth Analg v. 56 , n. 1, p. 55- 58, 1977.

CULLEN, L. K. Muscle relaxants and neuromuscular block, $p$. 337-364. In THURMON, J.C., TRANQUILI, W.J., BENSON, G. J. Lumb\&Jones' Veterinary Anesthesia, 3. ed., Lea \& Febiger, Blatimore, 1996.

GLEN, J. B., HUNTER, S. C., BLACKBURN, T. P. Interaction studies and other investigations of the pharmacology of propol (Dipivran). Postgraduate Medical Journal, v. 61, p. 714,1985 .

GOUDSOUZIAN, N. G, PARLSOE, C. P. Os novos relaxantes musculares em pediatria. Ver Bras Anestesiol v. 44, n. 2, p. 147-158, 1994.

HUGHES, R., CHAPPLE, D.J. 1981. The pharmacoloy of atracurium: a new competetive neuromuscular blocking agent. Br J Anaesth v. 53, p. 31-43, 1981.
JONES, R. S., HUNTER, J. M., UTTING, J. E. Neuromuscular blocking of atracurium in the dog and its reversal by neostigmine. Res Vet Sci v. 34, p. 173-176, 1983.

JONES, R. S. Muscle relaxants in canine anaesthesia 1: history and the drugs. J Small Anim Pract 33, p. 371-375, 1992.

Muscle relaxants in canine anaesthesia 2: history and the drugs. J Small Anim Pract 33, p. 423-429, 1992.

KLEIN, L. V. Neuromuscular blocking agents, p.134-153. In Short CE Principles and Practice of Veterinary Anesthesia, 1. ed., Williams \& Wilkins, Baltimore, 1987.

MAMA, K. R., STEFFEY, E. P. Evaluation of propofol as a general anesthetic for horses. Veterinary Surgery, v. 24, p. 188-194, 1995.

MARSHALL, B. E., LONGNECKER, D. E. General anesthetics, p. 307-330. In Hardman JG, Goodman Gilman A Goodman \& Gillman's The pharmacological basis of therapeutics, 9'ed., McGraw-Hill, Internacional, 1996.

McKELVY, D., HOLLINGSHEAD, K. W. Anesthetic agents and techniques, p. 119-162. In Small Animal Anesthesia: canine and feline practice, 1. ed., Mosby, Missouri, 1994.

TAYLOR, P. Agents acting at the neuromuscular junction and autonomic ganglia, p.177-197. In: Hardman, J.G., GOODMAN, Gilman. A Goodman \& Gillman's The pharmacological basis of therapeutics, 9. ed., McGraw-Hill, Internacional, 1996. 